\title{
Optimal Mobile Actuator/Sensor Network Motion Strategy for Parameter Estimation in a Class of Cyber Physical Systems
}

\author{
Christophe Tricaud and YangQuan Chen
}

\begin{abstract}
This paper introduces a framework to solve the problem of determining optimal sensors and actuators trajectories so as to estimate a set of unknown parameters in what constitutes a cyber-physical system (CPS). Given a distributed system's set of partial differential equation describing its dynamic behavior, the optimal steering of a team of sensors and actuators is obtained by minimizing the D-optimality criteria associated with the expected accuracy of the obtained parameter values. The problem is then reformulated into an optimal control one, whose solution can be computed by readily available commercial softwares. A numerical example is used to demonstrate the feasibility of the proposed method.
\end{abstract}

\section{INTRODUCTION}

\section{A. Literature Review}

The combination of physical systems and networks has brought to light a new generation of engineered systems: Cyber-Physical Systems (CPS) [1]. CPS is defined in [2] in the following way: "Computational thinking and integration of computation around the physical dynamic systems form CPS where sensing, decision, actuation, computation, networking and physical processes are mixed". CPS is foreseen to become a highly researched area in the years to come with its own conferences [3][4] and journals [5].

"Applications of CPS arguably have the potential to dwarf the 20-th century IT revolution" [6]. CPS applications can be found in medical devices and systems, patient monitoring devices, automotive and air traffic control, advanced automotive systems, process control, environmental monitoring, avionics, instrumentation, oil refineries, water usage control, cooperative robotics, manufacturing control, buildings, etc.

The first step when considering a CPS is to determine the dynamics of its "physical" part, i.e. the environment in which the sensors and actuators are going to operate. First by defining a matching mathematical model, and then by retrieving the values of the parameters of this model, the obtained accurate model allows for improved design of the CPS. In this paper, the parameter estimation process constitutes a CPS in itself as we are using a mobile actuatorsensor network for that purpose.

Corresponding author: Professor YangQuan Chen. T: 1(435)7970148; F: 1(435)7973054; E: yqchen@ieee.org; W: http://yangquan.chen.googlepages.com/.

This work is supported in part by the NSF International Research and Education in Engineering (IREE) grant $\sharp 0540179$.

C. Tricaud is with the Department of Electrical and Computer Engineering, Utah State University, 4120 Old Main Hill, Logan, Utah 84322-4120, USA c.tricaudeaggiemail.usu.edu

Y. Q. Chen is with the Department of Electrical and Computer Engineering, Utah State University, 4120 Old Main Hill, Logan, Utah 84322-4120, USA yqchen@ieee.org
The "model-analysis-design" process in dynamic systems control is fundamental in control engineering practice. In both physical and mathematical modeling, the parameter estimation is essential in successful control designs. A precise parameter estimation depends not only on "relevant" measurements and observations, but also on "rich" excitation of the system. These are all known concepts in system identification for finite dimensional systems [7]. In the present work, the system to be modeled is of distributed parameter nature (i.e. the states evolve along both time and space axes). Clearly, common finite-dimensional input-output relationships cannot be adopted to characterize the system and in turn, one needs to rely on partial differential equations (PDEs) for modeling.

However, determining where measurement should be done as well as where should the system be excited to increase the relevance of observations and measurements of the states of a distributed parameter system is not a straightforward task. One needs to consider the actuation capabilities as well as location of the sensors so that the gathered information best helps the parameter estimation. Therefore, it is a necessity to develop systematic approaches in order to increase the efficiency of PDE parameter estimators.

The problem of sensor location is not new as in, for example, review papers ([8][9]). However, the investigation on how to best excite the PDE system for optimal parameter estimation has not been attempted so far. This paper presents a framework for such optimal mobile actuation policy aiming at optimal parameter estimation of a class of distributed parameter systems.

In the field of mobile sensor trajectory planning in CPS, few approaches have been developed so far but a large collection of problems have been considered. Rafajówicz [10] investigates the problem using the determinant of the Fisher Information Matrix (FIM) associated with the parameters he wants to estimate. However, his results are more of an optimal time-dependent measure than a trajectory. In [9] and [11], Uciński reformulates the problem of time-optimal path planning into a state-constrained optimal-control one which allows the addition of different constraints on the dynamics of the sensor. Increased observability of the system is considered in [12]. In [13], Uciński tries to properly formulate and solve the time-optimal problem for moving sensors which observe the state of a DPS in order to estimate its parameters' value. In [14], [15], the detection of a moving source within of distributed system is considered for a sensor network. In [16], the state estimation of a distributed system is considered; mobile sensors steering policy is decided so as 
to imrpove the state estimate. In [17], both source detection and process estimation are combined into a single framework. In [18], the Turing's Measure of Conditioning is used to obtain optimal sensor trajectories. The problem is solved for heterogeneous sensors (i.e. with different measurement accuracies) in [19]. Limited power resource is considered in [20], [21]. In [22], Demetriou considers the optimal trajectories of mobile sensors in unison so as to improve state estimation. In [23], Song adds realistic constraints to the dynamics of the mobile sensor by considering a differential-drive mobile robot in the framework of the MASnet Project. In [24], a so-called "closed-loop" scheme was considered for the case where initial estimates of the system's parameters were inaccurate. This paper opened a window towards networking considerations as computations were not prior to the experiment anymore but "on-line". For the first time, actuators trajectories were considered and optimized in [25] for known sensor trajectories.

To the best of the authors' knowledge, this paper constitutes the first attempt to solve the problem where both sensors and actuators trajectories are optimized for parameter estimation in distributed parameter systems, hence the "cyber-physical" nature of the considered system. The equations describing the system's dynamics are assumed to be known but with some unknown parameters. A team of sensors and actuators is used to stimulate the system so that measurements from the sensors, possibly mobile, provide best information for parameter estimation.

\section{B. Problem Formulation for PDE Parameter Estimation}

Consider a distributed parameter system (DPS) described by the partial differential equation

$$
\frac{\partial y}{\partial t}=\mathcal{F}(\boldsymbol{x}, t, y, \boldsymbol{\theta}) \quad \text { in } \Omega \times T
$$

with initial and boundary conditions

$$
\begin{aligned}
\mathcal{B}(\boldsymbol{x}, t, y, \boldsymbol{\theta}) & =0 & & \text { on } \Gamma \times T, \\
y & =y_{0} & & \text { in } \Omega \times\{t=0\},
\end{aligned}
$$

where $y(\boldsymbol{x}, t)$ stands for the scalar state at a spatial point $\boldsymbol{x} \in \bar{\Omega} \subset \mathbb{R}^{n}$ and time instant $t \in \bar{T} . \Omega \subset \mathbb{R}^{n}$ is a bounded spatial domain with sufficiently smooth boundary $\Gamma$, and $T=$ $\left(0, t_{f}\right]$ is a bounded time interval. $\mathcal{F}$ is assumed to be a known well-posed, possibly nonlinear, differential operator which includes first- and second-order spatial derivatives and include terms for forcing inputs. $\mathcal{B}$ is a known operator acting on the boundary $\Gamma$ and $y_{0}=y_{0}(\boldsymbol{x})$ is a given function.

We assume that the state $y$ depends on the parameter vector $\boldsymbol{\theta} \in \mathbb{R}^{m}$ of unknown parameters to be determined from measurements made by $N$ static or moving pointwise sensors over the observation horizon $T$. We call $\boldsymbol{x}_{s}^{j}: T \rightarrow$ $\Omega_{\text {ad }}$ the position/trajectory of the $j$-th sensor, where $\Omega_{\text {ad }} \subset$ $\Omega \cup \Gamma$ is a compact set representing the domain where measurements are possible. The observations for the $j$-th sensor are assumed to be of the form

$$
z^{j}(t)=y\left(\boldsymbol{x}_{s}^{j}(t), t\right)+\varepsilon\left(\boldsymbol{x}_{s}^{j}(t), t\right), \quad t \in T, \quad j=1, \ldots, N,
$$

where $\epsilon$ represents the measurement noise assumed to be white, zero-mean, Gaussian and spatial uncorrelated with the following statistics

$$
\mathrm{E}\left\{\varepsilon\left(\boldsymbol{x}_{s}^{j}(t), t\right) \varepsilon\left(\boldsymbol{x}_{s}^{i}\left(t^{\prime}\right), t^{\prime}\right)\right\}=\sigma^{2} \delta_{j i} \delta(t-\tau),
$$

where $\sigma^{2}$ stands for the standard deviation of the measurement noise, $\delta_{i j}$ and $\delta(\cdot)$ are the Kronecker and Dirac delta functions, respectively.

With the above settings, similar to [9], the optimal parameter estimation problem is formulated as follows: Given the model (1)-(3) and the measurements $z^{j}$ from the sensors $\boldsymbol{x}_{s}^{j}$, $j=1, \ldots, N$, determine an estimate $\widehat{\boldsymbol{\theta}} \in \Theta_{\mathrm{ad}}\left(\Theta_{\mathrm{ad}}\right.$ being the set of admissible parameters) of the parameter vector which minimizes the generalized output least-squares fit-todata functional given by

$$
\widehat{\boldsymbol{\theta}}=\arg \min _{\boldsymbol{\vartheta} \in \Theta_{\mathrm{ad}}} \sum_{j=1}^{N} \int_{T}\left[z^{j}(t)-y\left(\boldsymbol{x}_{\boldsymbol{s}}^{j}(t), t ; \boldsymbol{\vartheta}\right)\right]^{2} \mathrm{~d} t
$$

where $y$ is the solution of (1)-(3) with $\boldsymbol{\theta}$ replaced by $\boldsymbol{\vartheta}$.

By observing (6), it is possible to foresee that the parameter estimate $\widehat{\boldsymbol{\theta}}$ depends on the number of sensors $N$ and the mobile sensor trajectories $x_{s}^{j}$. This fact triggered the research on the topic and explains why the literature so far focused on optimizing both the number of sensors and their trajectories. The intent was to select these design variables so as to produce best estimates of the system parameters after performing the actual experiment.

Note that, besides these explicit design variables there exists an implicit one that is the forcing input in (1). Therefore, in this paper, we assume that the forcing input is realized by a group of $M$ actuators, whose trajectories are to be optimized as well.

Since our approach is based on the methodology developed for optimal sensor location, we display it here as an introduction to the theory from [9] and [26]. In order to achieve optimal sensor location, some quality measure of sensor configurations based on the accuracy of the parameter estimates obtained from the observations is required. Such a measure is usually related to the concept of the Fisher Information Matrix (FIM), which is frequently referred to in the theory of optimal experimental design for lumped parameter systems [27]. Its inverse constitutes an approximation of the covariance matrix for the estimate of $\boldsymbol{\theta}$. Given the assumed statistics of the measurement noise, the FIM has the following representation [9], [28]:

$$
\boldsymbol{M}=\sum_{j=1}^{N} \int_{T} \boldsymbol{g}\left(\boldsymbol{x}_{s}^{j}(t), t\right) \boldsymbol{g}^{\top}\left(\boldsymbol{x}_{s}^{j}(t), t\right) \mathrm{d} t,
$$

where

$$
\boldsymbol{g}(\boldsymbol{x}, t)=\left.\nabla_{\boldsymbol{\vartheta}} y(\boldsymbol{x}, t ; \boldsymbol{\vartheta})\right|_{\boldsymbol{\vartheta}=\boldsymbol{\theta}^{0}}
$$

denotes the vector of the so-called sensitivity coefficients, $\boldsymbol{\theta}^{0}$ being a prior estimate to the unknown parameter vector $\theta$ [11], [9].

However, the FIM can hardly be used in an optimization as is. Therefore, it is necessary to maximize some scalar 
function $\Psi$ of the information matrix to obtain the optimal experiment setup. The introduction of the scalar criterion allows us to pose the sensor location problem as an optimization problem. Several choices for such a function can be found in the literature [29], [27], [30] and the most popular one is the D-optimality criterion

$$
\Psi[M]=-\log \operatorname{det}(\boldsymbol{M}) .
$$

Its use yields the minimal volume of the uncertainty ellipsoid for the estimates of the parameters. In this paper, only the D-optimality criterion is considered.

\section{OPTIMAL MEASUREMENT/ACTUATION PROBLEM}

\section{A. Mobile Sensor/Actuator Model}

We assume that both sensors and actuators are equipped on vehicles whose dynamics can be described by the following differential equation

$$
\dot{\boldsymbol{x}}_{x}^{j}(t)=\boldsymbol{f}_{x}\left(\boldsymbol{x}_{x}^{j}(t), \boldsymbol{u}_{x}^{j}(t)\right) \quad \text { a.e. on } T, \quad \boldsymbol{x}_{x}^{j}(0)=\boldsymbol{x}_{x 0}^{j},
$$

where $\boldsymbol{x}$ can stand for two different categories. The first being $s$ for sensors and the second being $a$ for actuators.

With this nomenclature, the function $\boldsymbol{f}_{x}\left(\boldsymbol{f}_{s}: \mathbb{R}^{N} \times \mathbb{R}^{r_{s}} \rightarrow\right.$ $\mathbb{R}^{N}$ for sensors, $\boldsymbol{f}_{a}: \mathbb{R}^{M} \times \mathbb{R}^{r_{a}} \rightarrow \mathbb{R}^{M}$ for actuators) has to be continuously differentiable, the vector $\boldsymbol{x}_{x 0}^{j}\left(\boldsymbol{x}_{s 0}^{j} \in \mathbb{R}^{N}\right.$ for sensors, $\boldsymbol{x}_{a 0}^{j} \in \mathbb{R}^{M}$ for actuators) represents the initial disposition of the $j$-th sensor/actuator, and $\boldsymbol{u}_{x}\left(\boldsymbol{u}_{s}: T \rightarrow\right.$ $\mathbb{R}^{r_{s}}$ for sensors, $\boldsymbol{u}_{a}: T \rightarrow \mathbb{R}^{r_{a}}$ for actuators) is a measurable control function satisfying the following inequality

$$
\boldsymbol{u}_{x l} \leq \boldsymbol{u}_{x}(t) \leq \boldsymbol{u}_{x u} \quad \text { a.e. on } T,
$$

for some known constant vectors $\boldsymbol{u}_{x l}$ and $\boldsymbol{u}_{x u}$. Let us introduce,

$$
\boldsymbol{s}(t)=\left(\boldsymbol{x}_{s}^{1}(t), \boldsymbol{x}_{s}^{2}(t), \ldots, \boldsymbol{x}_{s}^{N}(t), \boldsymbol{x}_{a}^{1}(t), \ldots, \boldsymbol{x}_{a}^{M}(t)\right)^{T},
$$

where $\boldsymbol{x}_{s}^{j}: T \rightarrow \Omega_{\text {sad }}$ is the trajectory of the $j$-th sensor and $\boldsymbol{x}_{a}^{k}: T \rightarrow \Omega_{a a d}$ is the trajectory of the $k$-th actuator. We assume that all the mobile nodes equipped with sensors are confined within an admissible region $\Omega_{\text {sad }}$ (a given compact set) where the measurements are possible and reciprocally that all mobile nodes equipped with actuators are restrained in a domain $\Omega_{a a d}$ where actuation can be achieved. Considering the general index $x$ defined earlier, $\Omega_{x a d}$ can be conveniently defined as

$$
\Omega_{x a d}=\left\{\boldsymbol{x}_{x} \in \Omega: b_{x i}\left(\boldsymbol{x}_{x}\right)=0, i=1, \ldots, I\right\},
$$

where the $b_{x i}$ functions are known continuously differentiable functions. That is to say that the following constraints have to be satisfied:

$$
h_{i j}(\boldsymbol{s}(t))=b_{x} i\left(\boldsymbol{x}_{x}^{j}(t)\right) \leq 0, \forall t \in T,
$$

where $1 \leq i \leq I$ and $1 \leq j \leq(N+M)$. For simpler notation, we reformulate the conditions described in (14) in the following way

$$
\gamma_{l}(s(t)) \leq 0, \forall t \in T
$$

where $\gamma_{l}, l=1, \ldots, \nu$ tally with (14), $\nu=I \times(N+M)$. It would be possible to consider additional constraints on the path of the vehicles such as specific dynamics, collision avoidance, communication range maintenance and any other conceivable constrains.

The actuation function for the $k$-th mobile actuator is assumed to depend on the actuator's position as reflected by the following definition

$$
\mathcal{F}_{k}(\boldsymbol{x}, t)=\mathcal{G}_{k}\left(\boldsymbol{x}, \boldsymbol{x}_{a}^{k}, t\right)
$$

\section{B. Problem Definition}

The measurement/actuation problem can be defined by reformulating (1) in the following way

$$
\frac{\partial y}{\partial t}=\mathcal{F}(\boldsymbol{x}, t, y, \boldsymbol{\theta})+\sum_{k=1}^{M} \mathcal{F}_{k}(\boldsymbol{x}, t) \quad \text { in } \Omega \times T,
$$

initial and boundary conditions remain unchanged. $\mathcal{F}$ may still include forcing inputs terms unrelated to the actuators.

Based on this updated definition, the FIM associated with the problem is given by the following new representation

$$
\boldsymbol{M}(\boldsymbol{s})=\sum_{k=1}^{M} \int_{T} \boldsymbol{h}\left(\boldsymbol{x}_{a}^{k}(t), t\right) \mathrm{d} t
$$

where for the $k$-th actuator

$$
\boldsymbol{h}\left(\boldsymbol{x}_{a}^{k}(t), t\right)=\sum_{j=1}^{N} \boldsymbol{g}\left(\boldsymbol{x}_{a}^{k}(t), \boldsymbol{x}_{s}^{j}(t), t\right) \boldsymbol{g}^{\top}\left(\boldsymbol{x}_{a}^{k}(t), \boldsymbol{x}_{s}^{j}(t), t\right)
$$

and

$$
\boldsymbol{g}\left(\boldsymbol{x}_{a}^{k}(t), \boldsymbol{x}(t), t\right)=\left.\int_{0}^{t} \nabla_{\boldsymbol{\vartheta}} y(\boldsymbol{x}(\tau), \tau ; \boldsymbol{\vartheta})\right|_{\boldsymbol{\vartheta}=\boldsymbol{\theta}^{0}} \mathrm{~d} \tau .
$$

In (20), $y$ is the solution of (17) for $\mathcal{F}_{k}(\boldsymbol{x}, \tau)=$ $\mathcal{G}_{i}\left(\boldsymbol{x}, \boldsymbol{x}_{a}^{i}, \tau\right) \delta(t-\tau)$ for all $k \in[1, M]$.

The purpose of the optimal measurement/actuation problem is to determine the forces (controls) applied to each vehicle (conveying either a sensor or an actuator), which minimize the design criterion $\Psi(\cdot)$ defined on the FIMs of the form (7), which are determined unequivocally by the corresponding trajectories, subject to constraints on the magnitude of the controls and induced state constraints. To increase the degree of optimality, our approach considers $s_{0}$ as a control parameter vector to be optimized in addition to the control function $\boldsymbol{u}=\left[\boldsymbol{u}_{s} \boldsymbol{u}_{a}\right]^{T}$.

Given the above formulation we can cast the optimal measurement/actuation policy problem as the following optimization problem: Find the pair $\left(\boldsymbol{s}_{0}, \boldsymbol{u}\right)$ which minimizes

$$
J\left(\boldsymbol{s}_{0}, \boldsymbol{u}\right)=\Phi[\boldsymbol{M}(\boldsymbol{s})]
$$

over the set of feasible pairs

$$
\begin{aligned}
\mathcal{P}= & \left\{\left(s_{0}, u\right) \mid u: T \rightarrow \mathbb{R}^{r} \quad\right. \text { is measureable, } \\
& \left.u_{l} \leq u(t) \leq u_{u} \text { a.e. on } T, \boldsymbol{s}_{0} \in \Omega_{\text {sad }} \times \Omega_{\text {aad }}\right\},
\end{aligned}
$$

subject to the constraint (15). 
The solution to this problem can hardly have an analytical solution. It is therefore necessary to rely on numerical techniques to solve the problem. A wide variety of techniques are available [31]. However, the problem can be reformulated as a classical Mayer problem where the performance index is defined only via terminal values of state variables.

\section{OPTIMAL CONTROL FORMULATION}

In this section, the problem is converted into a canonical optimal control one making possible the use of existing optimal control problems solvers.

To simplify our presentation, we define the function svec $: \mathbb{S}^{m} \rightarrow \mathbb{R}^{m(m+1) / 2}$, where $\mathbb{S}^{m}$ denotes the subspace of all symmetric matrices in $\mathbb{R}^{m \times m}$ that takes the lower triangular part (the elements only on the main diagonal and below) of a symmetric matrix $A$ and stacks them into a vector $\boldsymbol{a}$ :

$$
\begin{aligned}
\boldsymbol{a}= & \operatorname{svec}(A) \\
= & \operatorname{col}\left[A_{11}, A_{21}, \ldots, A_{m 1}, A_{22}, \ldots\right. \\
& \left.A_{32}, \ldots, A_{m 2}, \ldots, A_{m m}\right] .
\end{aligned}
$$

Reciprocally, let $A=\operatorname{Smat}(\boldsymbol{a})$ be the symmetric matrix such that $\operatorname{svec}(\operatorname{Smat}(\boldsymbol{a}))=\boldsymbol{a}$ for any $\boldsymbol{a} \in \mathbb{R}^{m(m+1) / 2}$.

Consider the matrix-valued function

$$
\Pi(\boldsymbol{s}(t), t)=\sum_{k=1}^{M} \boldsymbol{h}\left(\boldsymbol{x}_{a}^{k}(t), t\right) .
$$

Setting $r: T \rightarrow \mathbb{R}^{m(m+1) / 2}$ as the solution of the differential equations

$$
\dot{\boldsymbol{r}}(t)=\operatorname{svec}(\Pi(\boldsymbol{s}(t), t)), \quad r(0)=0,
$$

we obtain

$$
M(s)=\operatorname{Smat}\left(\boldsymbol{r}\left(t_{f}\right)\right)
$$

i.e., minimization of $\Phi[M(s)]$ thus reduces to minimization of a function of the terminal value of the solution to (26). Introducing an augmented state vector

$$
\boldsymbol{q}(t)=\left[\begin{array}{c}
\boldsymbol{s}(t) \\
\boldsymbol{r}(t)
\end{array}\right]
$$

we obtain

$$
\boldsymbol{q}_{0}=\boldsymbol{q}(0)=\left[\begin{array}{c}
\boldsymbol{s}_{0} \\
\mathbf{0}
\end{array}\right]
$$

Then the equivalent canonical optimal control problem consists in finding a pair $\left(\boldsymbol{q}_{0}, \boldsymbol{u}\right) \in \overline{\mathcal{P}}$ which minimizes the performance index

$$
\bar{J}\left(\boldsymbol{q}_{0}, \boldsymbol{u}\right)=\phi\left(\boldsymbol{q}\left(t_{f}\right)\right)
$$

subject to

$$
\left\{\begin{aligned}
\dot{\boldsymbol{q}}(t) & =\phi(\boldsymbol{q}(t), \boldsymbol{u}(t), t) \\
\boldsymbol{q}(0) & =\boldsymbol{q}_{0} \\
\bar{\gamma}_{l}(\boldsymbol{q}(t)) & \leq 0
\end{aligned}\right.
$$

where

$$
\begin{aligned}
\overline{\mathcal{P}}= & \left\{\left(\boldsymbol{q}_{0}, \boldsymbol{u}\right) \mid \boldsymbol{u}: T \rightarrow \mathbb{R}^{r} \quad\right. \text { is measurable } \\
& \left.\boldsymbol{u}_{l} \leq \boldsymbol{u}(t) \leq \boldsymbol{u}_{u} \text { a.e. on } T, \boldsymbol{s}_{0} \in \Omega_{a d}^{M}\right\}
\end{aligned}
$$

and

$$
\begin{aligned}
\phi(\boldsymbol{q}, \boldsymbol{u}, t) & =\left[\begin{array}{c}
f(\boldsymbol{s}(t), \boldsymbol{u}(t)) \\
\operatorname{svec}(\Pi(\boldsymbol{s}(t), t))
\end{array}\right], \\
\bar{\gamma}_{l}(\boldsymbol{q}(t)) & =\gamma_{l}(\boldsymbol{s}(t)) .
\end{aligned}
$$

The above problem in canonical form can be solved using one of the existing packages for numerically solving dynamic optimization problems, such as RIOTS_95 [32], DIRCOL [33] or MISER [34]. We chose RIOTS_95, which is designed as a MATLAB toolbox written mostly in $\mathrm{C}$ and runs under Windows 98/2000/XP and Linux. The theory behind RIOTS_95 can be found in [35].

\section{AN ILLUSTRATIVE EXAMPLE}

In this section, we use a demonstrative example to illustrate our method. We consider the two-dimensional diffusion equation

$$
\frac{\partial y}{\partial t}=\nabla \cdot(\kappa \nabla y)+\sum_{k=1}^{M} F_{k}
$$

for $\boldsymbol{x}=\left[\begin{array}{ll}x_{1} & x_{2}\end{array}\right]^{T} \in \Omega=(0,1)^{2}$ and $t \in[0,1]$, subject to homogeneous zero initial and Dirichlet boundary conditions. The spatial distribution of the diffusion coefficient is assumed to have the form

$$
\kappa\left(x_{1}, x_{2}\right)=\theta_{1}+\theta_{2} x_{1}+\theta_{3} x_{2} .
$$

In our example, we select the initial estimates of the parameter values as $\theta_{1}^{0}=0.1, \theta_{2}^{0}=-0.05$ and $\theta_{3}^{0}=0.2$, which are assumed to be nominal and known prior to the experiment. The actuation function is

$$
F_{k}\left(\boldsymbol{x}, \boldsymbol{x}_{a}^{k}, t\right)=10 e^{-50\left(\left(x_{a 1}^{k}-x_{1}\right)^{2}+\left(x_{a 2}^{k}-x_{2}\right)^{2}\right)} .
$$

where $\boldsymbol{x}_{a}^{i}=\left[\begin{array}{ll}x_{a 1}^{i} & x_{a 2}^{i}\end{array}\right]^{T}$. The dynamics of the mobile actuators follow the simple model

$$
\dot{\boldsymbol{x}}_{a}^{k}(t)=\boldsymbol{u}_{a}^{k}(t), \quad \boldsymbol{x}_{a}^{k}(0)=\boldsymbol{x}_{a 0}^{k},
$$

and additional constraints

$$
\left|u_{a i}^{k}(t)\right| \leq 0.7, \quad \forall t \in T, \quad k=1, \ldots, M, \quad i=1, \ldots, 2 .
$$

The dynamics of the mobile sensors follow the same model

$$
\dot{\boldsymbol{x}}_{s}^{j}(t)=\boldsymbol{u}_{s}^{j}(t), \quad \boldsymbol{x}_{s}^{j}(0)=\boldsymbol{x}_{s 0}^{j},
$$

and additional constraints

$$
\left|u_{s i}^{j}(t)\right| \leq 0.7, \quad \forall t \in T, \quad j=1, \ldots, N, \quad i=1, \ldots, 2 .
$$

Our goal is to design their trajectories so as to obtain possibly the best estimates of $\theta_{1}, \theta_{2}$ and $\theta_{3}$.

The determination of the Fisher information matrix for a given experiment requires the knowledge of the vector of the sensitivity coefficients $\boldsymbol{g}=\operatorname{col}\left[g_{1}, g_{2}, g_{3}\right]$ along sensor trajectories. The FIM can be obtained using the direct 
differentiation method [9] by solving the following set of PDEs:

$$
\begin{aligned}
\frac{\partial y}{\partial t} & =\nabla \cdot(\kappa \nabla y)+\sum F_{k}, \\
\frac{\partial g_{1}}{\partial t} & =\nabla \cdot \nabla y+\nabla \cdot\left(\kappa \nabla g_{1}\right), \\
\frac{\partial g_{2}}{\partial t} & =\nabla \cdot\left(x_{1} \nabla y\right)+\nabla \cdot\left(\kappa \nabla g_{2}\right), \\
\frac{\partial g_{3}}{\partial t} & =\nabla \cdot\left(x_{2} \nabla y\right)+\nabla \cdot\left(\kappa \nabla g_{3}\right),
\end{aligned}
$$

in which the first equation represents the original state equation and the next three equations are obtained from the differentiation of the first equation with respect to the parameters $\theta_{1}, \theta_{2}$ and $\theta_{3}$, respectively. The initial and Dirichlet boundary conditions for all the four equations are homogeneous.

The strategy is tested on a simple team of one sensor and one actuator. In order to avoid getting stuck in a local minimum, computations were repeated several times from different initial solutions. Fig. 1 present the resulting trajectories for the run where the initial solutions lead to the best results (minimal value of the D-optimality criteria). Steering signals for both sensor and actuator are displayed in Fig. 2-5. Resulting trajectories for two sensors and one actuator are given in Fig. 6, and three sensors and one actuator in Fig. 7. Sensor trajectories are displayed in blue while actuator trajectories are red.

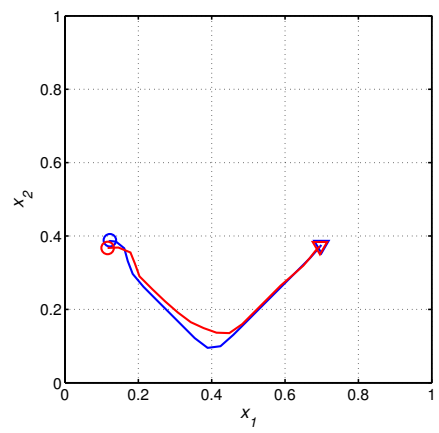

Fig. 1. D-Optimal trajectories of a team of one sensor and one actuator. The initial positions are marked with open circles and the final positions are designated by triangles

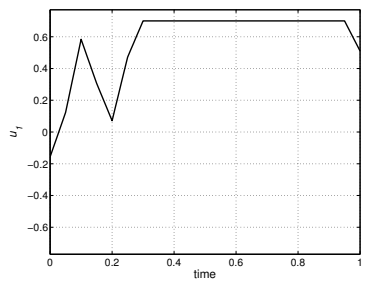

Fig. 2. Optimal control of the sensor with respect to the $x_{1}$-axis

\section{CONCLUSION}

We introduced the optimal measurement/actuation framework for parameter identification in a cyber-physical system

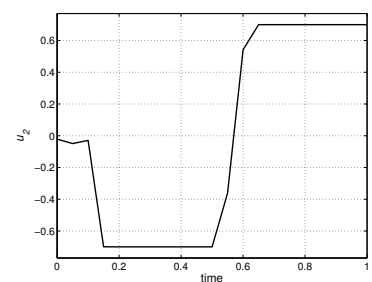

Fig. 3. Optimal control of the sensor with respect to the $x_{2}$-axis

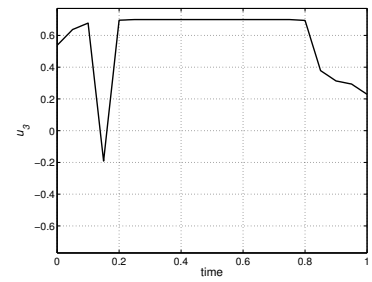

Fig. 4. Optimal control of the actuator with respect to the $x_{1}$-axis

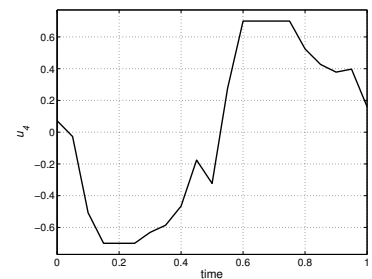

Fig. 5. Optimal control of the actuator with respect to the $x_{2}$-axis

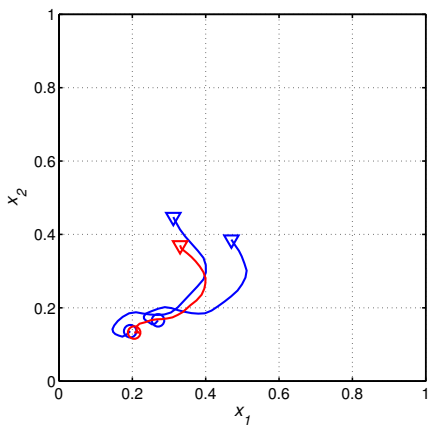

Fig. 6. D-Optimal trajectories of a team of two sensors and one actuator.

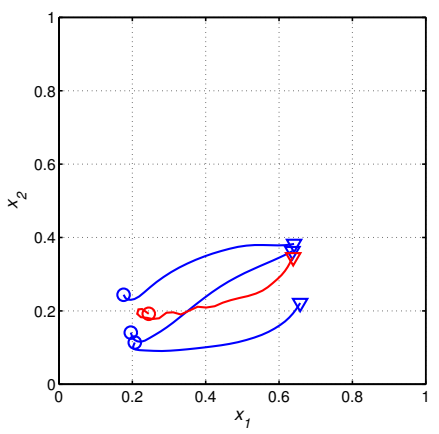

Fig. 7. D-Optimal trajectories of a team of three sensors and one actuator. 
constituted of mobile sensors and actuators behaving in a distributed parameter systems. The problem was formulated as an optimization problem using the concept of the Fisher information matrix. The problem was then reformulated into an optimal control one. With the help of the Matlab PDE toolbox for the system simulations and RIOTS_95 Matlab toolbox for solving the optimal control problem, we successfully obtained the optimal solutions for an illustrative example. Combined with the on-line scheme introduced in [24], this research represents a realistic example of CPS. Mobile sensors and actuators are communicating to achieve the parameter estimation of the physical system they are monitoring/stimulating. An exciting application consists in center-pivot operations, where our research center has a project of using camera-equipped unmanned air vehicles for soil-moisture measurement combined with irrigators to stimulate the farming field. Thanks to this framework, an accurate model of the soil dynamics can be derived and water savings can be obtained via optimal operations of the centerpivot.

\section{REFERENCES}

[1] C. S. Group, "Cyber-physical systems executive summary," in Cyber-Physical Systems Summit, 2008. [Online]. Available: http://varma.ece.cmu.edu/summit/CPS-Executive-Summary.pdf

[2] Y. Q. Chen, "Mobile actuator/sensor networks (MAS-net) for cyber-physical systems," USU ECE 6800 Graduate Colloquium, Fall 2008, September 2008. [Online]. Available: http://www.neng.usu.edu/classes/ece/6800/

[3] "NSF workshop on cyber-physical systems," October 2006. [Online]. Available: http://warma.ece.cmu.edu/cps/

[4] "The first international workshop on cyber-physical systems," June 2008. [Online]. Available: http://www.qhdctc.com/wcps2008/

[5] H. Gill, W. Zhao, and T. Znati, "Call for papers for a special issue of on distributed cyber physical systems," IEEE Transactions on Parallel and Distributed Systems (TPDS), vol. 19, no. 8, pp. 1150 - 1151, August 2008.

[6] E. A. Lee, "Computing foundations and practice for cyber-physical systems: A preliminary report," University of California, Berkeley, Tech. Rep. UCB/EECS-2007-72, May 2007. [Online]. Available: http://chess.eecs.berkeley.edu/pubs/306.html

[7] L. Ljung, System Identification Toolbox TM7 User's guide, The MathWorks ${ }^{\mathrm{TM}}$, 2008. [Online]. Available: http://www.mathworks.com/products/sysid/

[8] C. S. Kubrusly and H. Malebranche, "Sensors and controllers location in distributed systems a survey," Automatica, vol. 21, no. 2, pp. 117$128,1985$.

[9] D. Uciński, Optimal Measurement Methods for Distributed-Parameter System Identification, F. Boca Raton, Ed. CRC Press, 2005.

[10] E. Rafajówicz, "Optimum choice of moving sensor trajectories for distributed parameter system identification," International Journal of Control, vol. 43, no. 5, p. 14411451, 1986.

[11] D. Uciński, "Optimal sensor location for parameter estimation of distributed processes," International Journal of Control, vol. 73, no. 13, 2000.

[12] D. Uciński and M. A. Demetriou, "An approach to the optimal scanning measurement problem using optimum experimental design," in Proceedings of the 2004 American Control Conference (ACC04), June-July 2004.

[13] D. Uciński and Y. Chen, "Time-optimal path planning of moving sensors for parameter estimation of distributed systems," Proc. 44th IEEE Conference on Decision and Control, and the European Control Conference, Seville, Spain, 2005.

[14] M. A. Demetriou, "Power management of sensor networks for detection of a moving source in 2-D spatial domains," in Proceedings of the 2006 American Control Conference (ACC06), June 2006.

[15] —, "Detection and containment policy of moving source in 2-D diffusion processes using sensor/actuator network," in Proceedings of the 2007 European Control Conference (ECC07), July 2007.
[16] I. Hussein and M. A. Demetriou, "Estimation of distributed processes using mobile spatially distributed sensors," in Proceedings of the 2007 American Control Conference (ACC07), July 2007.

[17] M. A. Demetriou, "Process estimation and moving source detection in 2-D diffusion processes by scheduling of sensor networks," in Proceedings of the 2007 American Control Conference (ACC07), July 2007.

[18] D. Uciński and Y. Chen, "Sensor motion planning in distributed parameter systems using Turing's measure of conditioning," in Proc. 45th IEEE Conference on Decision and Control, San Diego, CA, 2006, published on CD-ROM.

[19] C. Tricaud, M. Patan, D. Uciński, and Y. Chen, "D-optimal trajectory design of heterogeneous mobile sensors for parameter estimation of distributed systems," in Proc. 2008 American Control Conference, Seattle, Washington, USA, 2008, published on CD-ROM.

[20] M. Patan, C. Tricaud, and Y. Chen, "Resource-constrained sensor routing for parameter estimation of distributed systems," in Proc. 17th IFAC World Congress, Seoul, Korea, 2008, published on CD-ROM.

[21] D. Uciński and M. A. Demetriou, "Resource-constrained sensor routing for optimal observation of distributed parameter systems," in Proceedings of the 18th International Symposium on Mathematical Theory of Networks and Systems (MTNSO8). SIAM, July 2008.

[22] M. A. Demetriou, "Distributed parameter methods for moving sensor networks in unison," in Proceedings of the 2008 American Control Conference (ACCO8), June 2008.

[23] Z. Song, Y. Chen, J. Liang, and D. Uciński, "Optimal mobile sensor motion planning under nonholonomic constraints for parameter estimation of distributed parameter systems," IEEE/RSJ International Conference on Intelligent Robots and Systems, Edmonton, Alberta, Canada, 2005.

[24] C. Tricaud and Y. Q. Chen, "Optimal mobile sensing policy for parameter estimation of distributed parameter systems finite horizon closedloop solution," in Proceedings of the 18th International Symposium on Mathematical Theory of Networks and Systems (MTNSO8). SIAM, July 2008.

[25] C. Tricaud and Y. Chen, "Optimal mobile actuation policy for parameter estimation of distributed parameter systems," in Proceedings of the 18th International Symposium on Mathamatical Theory of Networks and Systems (MTNSO8). SIAM, July 2008.

[26] M. Patan, "Optimal observation strategies for parameter estimation of distributed systems," Ph.D. dissertation, University of Zielona Góra, Zielona Góra, Poland, 2004.

[27] V. V. Fedorov and P. Hackl, Model-Oriented Design of Experiments, ser. Lecture Notes in Statistics. New York: Springer-Verlag, 1997.

[28] Z. H. Quereshi, T. S. Ng, and G. C. Goodwin, "Optimum experimental design for identification of distributed parameter systems," International Journal of Control, vol. 31, no. 1, pp. 21-29, 1980.

[29] É. Walter and L. Pronzato, Identification of Parametric Models from Experimental Data, ser. Communications and Control Engineering. Berlin: Springer-Verlag, 1997.

[30] A. C. Atkinson and A. N. Donev, Optimum Experimental Designs. Oxford: Clarendon Press, 1992.

[31] E. Polak, Optimization. Algorithms and Consistent Approximations, ser. Applied Mathematical Sciences. New York: Springer-Verlag, 1997.

[32] A. L. Schwartz, E. Polak, and Y. Chen, A Matlab Toolbox for Solving Optimal Control Problems. Version 1.0 for Windows, May 1997. [Online]. Available: http://www.schwartz-home.com/ adam/RIOTS/

[33] O. von Stryk, User's Guide for DIRCOL, a Direct Collocation Method for the Numerical Solution of Optimal Control Problems. Version 2.1, Fachgebiet Simulation und Systemoptimierung, Technische Universität Darmstadt, Nov. 1999. [Online]. Available: http://www.sim.informatik.tu-darmstadt.de/index/leftnav.html.en

[34] L. S. Jennings, M. E. Fisher, K. L. Teo, and C. J. Goh, MISER 3: Optimal Control Software, Version 2.0. Theory and User Manual, Department of Mathematics, University of Western Australia, Nedlands, 2002. [Online]. Available: http://www.cado.uwa.edu.au/miser/

[35] A. L. Schwartz, "Theory and implementation of numerical methods based on Runge-Kutta integration for solving optimal control problems," Ph.D. dissertation, University of California, Berkeley, 1996. 